EPJ manuscript No.

(will be inserted by the editor)

\title{
A continuum model for the flow of thin liquid films over intermittently chemically patterned surfaces
}

\author{
J.E. Sprittles ${ }^{\mathrm{a}}$ and Y.D. Shikhmurzaev ${ }^{\mathrm{b}}$ \\ School of Mathematics, University of Birmingham, Birmingham, B15 2TT, UK.
}

\begin{abstract}
It is known from both experiments and molecular dynamics simulations that chemically patterning a solid surface has an effect on the flow of an adjacent liquid. This fact is in stark contrast with predictions of classical fluid mechanics where the no-slip boundary condition is insensitive to the chemistry of the solid substrate. It has been shown that the influence on the flow caused by a steep change in the wettability of the solid substrate can be described in the framework of continuum mechanics using the interface formation theory. The present work extends this study to the case of intermittent patterning. Results show that variations in wettability of the substrate can significantly affect the flow, especially of thin films, which may have applications to the design of microfluidic devices.
\end{abstract}

\section{Introduction}

Flows of liquids on small length scales, characterised by high surface-to-volume ratios, are of increasing interest in many emerging technologies 11. The correct description of the physics at liquid-solid interfaces is imperative to successful modelling of such phenomena 2]. Particular interest lies in how modification of the chemical properties of the solid substrate, most importantly variation in its wettability, may affect the flow of an adjacent fluid. In classical fluid mechanics, the no-slip boundary condition is applied on a solid substrate irrespective of its chemical properties, whereas molecular dynamics simulations $[3$ and experiments 4 have shown that deviations from this condition can be significant, even for chemically homogenous substrates, when considering flows on small length scales. The conventional generalisation of no-slip is the Navier slip condition [5], which states that slip (a non-zero difference between the tangential velocities of the fluid and substrate) is proportional to the shear stress acting on the liquid-solid interface from the fluid. Using the coefficient of proportionality as an adjustable parameter, one can describe flow over chemically homogenous solid substrates in situations where slip is important (e.g., [6]). However, the Navier condition is a dynamic boundary condition and as such it is unrelated to the solid's wettability, which can be defined in the absence of motion, for example in terms of the static contact angle between a free surface and the solid substrate. Consequently, the Navier condition offers no framework that would allow one to incorporate the solid's wettability and, in this sense, has no advantages over the no-slip condition.

It has been shown [7] that the interface formation theory 8 that has previously been applied to describe dynamic wetting and some other phenomena 91011 can be used, without any alterations, to study the effect of a variation in wettability of the solid on the flow. In the present work, we consider a particular application of the theory in the situation where the pattern on the solid substrate consists of an array of stripes of differing wettabilities.

a e-mail: sprittlj@maths.bham.ac.uk

b e-mail: yulii@for.mat.bham.ac.uk 


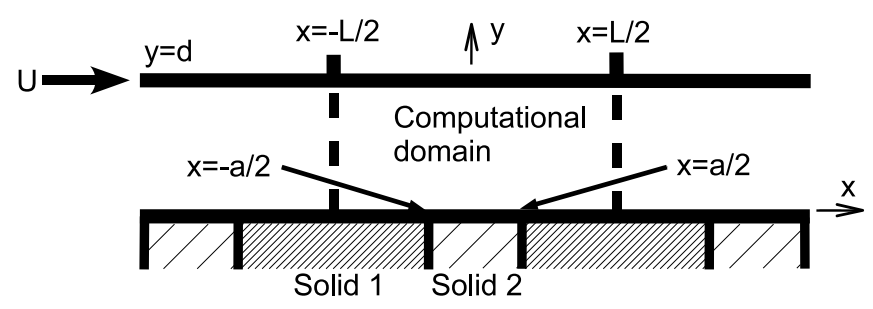

Fig. 1. Problem setup.

\section{Problem formulation}

Consider the steady two-dimensional flow of an incompressible Newtonian liquid between two solid surfaces (Fig. 10 where the upper surface is chemically homogenous and moves parallel to itself with velocity $\mathbf{u}=(u, v)=(U, 0)$, whilst the lower surface is chemically patterned and remains stationary. The pattern on the lower surface consists of intermittent stripes of solid 1, with width $L-a$, and solid 2, with width $a$. It is assumed that both surfaces are perfectly smooth. The wettabilities of solid 1 and solid 2 are characterised by the contact angles, $\theta_{i}$ $(i=1,2)$, which a liquid-gas free surface would form with solid $i$.

In the bulk the flow parameters satisfy the Navier-Stokes equations,

$$
\nabla \cdot \mathbf{u}=0, \quad \rho \mathbf{u} \cdot \nabla \mathbf{u}=-\nabla p+\mu \nabla^{2} \mathbf{u},
$$

where $p$ is the pressure, $\rho$ is the density and $\mu$ is the viscosity of the fluid. On both surfaces we apply the boundary conditions provided by the interface formation theory [8]:

$$
\begin{gathered}
\sigma=\gamma\left(\rho_{(0)}^{s}-\rho^{s}\right) \\
\mu\left(\frac{\partial u}{\partial y}+\frac{\partial v}{\partial x}\right)+\frac{1}{2}\left(\frac{d \sigma}{d x}+\frac{\gamma \rho^{s}}{\rho_{e}^{s}} \frac{d \rho_{e}^{s}}{d x}\right)=\beta u, \quad \rho v=\frac{\rho^{s}-\rho_{e}^{s}}{\tau} \\
\frac{d\left(\rho^{s} v^{s}\right)}{d x}=-\frac{\rho^{s}-\rho_{e}^{s}}{\tau} \\
v^{s}=\frac{1}{2} u+\alpha\left(\frac{d \sigma}{d x}+\frac{\gamma \rho^{s}}{\rho_{e}^{s}} \frac{d \rho_{e}^{s}}{d x}\right) .
\end{gathered}
$$

A detailed explanation of the process of interface formation together with a derivation of the equations and applications of the theory are given in several works (e.g. 8910]) so that here we will briefly recapitulate only the important points.

At constant temperature the state of the liquid-solid interface, i.e. physically, a microscopic layer of liquid adjacent to the solid surface, is characterised by the surface density $\rho^{s}$ of liquid in this layer. In equilibrium one has $\rho^{s}=\rho_{e}^{s}$ and the surface tension $\sigma\left(\rho_{e}^{s}\right)=\sigma_{e}$, where the equilibrium values $\rho_{e}^{s}$ and $\sigma_{e}$ are different for solids of different wettability. The simplest equation of state accounting for the influence of $\rho^{s}$ on $\sigma$ is given by (2). Instead of no-slip one now has (3), where the first equation is the generalised Navier condition given here for the case of a variable $\rho_{e}^{s}$ and the second describes the mass exchange between the bulk and the liquid-solid interface. This mass exchange is also accounted for in the mass balance equation for the interface (4). Finally, equation (5) describes how the velocity in the interface $v^{s}$ depends on the bulk velocity $u$ evaluated on the liquid-facing side of the liquid-solid interface and the surface tension gradient. The parameters $\alpha$ and $\beta$ characterise the response of the interface to surface tension gradients and external torque, respectively; $\gamma$ is associated with the inverse compressibility of the fluid; $\rho_{(0)}^{s}$ is the surface density corresponding to zero surface tension; $\tau$ is the surface tension relaxation time; in the simplest variant of the theory these are all treated as material constants.

The terms including $d \rho_{e}^{s} / d x$ in (3) and (5) describe the surface force which ensures that, in the case of a variable $\rho_{e}^{s}$ (and hence $\sigma_{e}$ ), there is no perpetual motion. Once the outer flow 
drives the matter in the liquid-solid interface along a solid surface whose wettability varies, the surface tension gradient in (3) and (5) becomes unbalanced triggering the interface formation process which in its turn affects the flow that caused it.

The last step now is to specify the wettability pattern of the lower surface, i.e. prescribe the dependence of $\rho_{e}^{s}$ on coordinates along it and the value of $\rho_{e}^{s}$ on the upper surface. From the viewpoint of fluid mechanics, the junction between solid 1 and solid 2 is associated with a discontinuity in the properties of the substrate and, therefore, requires special handling. In order to avoid this, we consider a transition region in which the equilibrium surface density varies smoothly, from its value $\rho_{1 e}^{s}$ on solid 1 to value $\rho_{2 e}^{s}$ on solid 2 (or vice-versa), across a finite distance $l$. This can be modelled, for example, using

$$
\rho_{e}^{s}=\rho_{1 e}^{s}+\frac{1}{2}\left(\rho_{2 e}^{s}-\rho_{1 e}^{s}\right)\{\tanh [(a / 2+x) / l]+\tanh [(a / 2-x) / l]\} .
$$

On the upper solid we assume $\rho_{e}^{s} \equiv \rho_{1 e}^{s}$.

Using the Young equation, $\sigma\left(\rho_{i e}^{s}\right)=-\sigma_{l g} \cos \theta_{i}$, where $\sigma_{l g}$ is the surface tension of the liquidgas free surface, and the equation of state (2) one can relate $\rho_{1 e}^{s}$ (or $\rho_{2 e}^{s}$ ) with the equilibrium contact angle $\theta_{1}$ (or $\theta_{2}$ ) which the free surface would form with solid 1 (or 2 ):

$$
\rho_{i e}^{s}=\rho_{(0)}^{s}+\gamma^{-1} \sigma_{l g} \cos \theta_{i}, \quad(i=1,2) .
$$

The contact angle is the conventional and convenient measure of the solid surface wettability with respect to a given fluid, and in what follows we use $\theta_{i}$ instead of $\rho_{i e}^{s}$.

Due to the periodicity of the problem it is convenient to use periodic boundary conditions

$$
\begin{gathered}
\left.\mathbf{u}\right|_{x=-L / 2}=\left.\mathbf{u}\right|_{x=L / 2},\left.\quad \frac{\partial \mathbf{u}}{\partial x}\right|_{x=-L / 2}=\left.\frac{\partial \mathbf{u}}{\partial x}\right|_{x=L / 2}, \\
\left.\rho^{s}\right|_{x=-L / 2}=\left.\rho^{s}\right|_{x=L / 2},\left.\quad v^{s}\right|_{x=-L / 2}=\left.v^{s}\right|_{x=L / 2} .
\end{gathered}
$$

Equations (1)-(6), (8)-(9) now fully specify our problem.

\section{Results}

The problem has been solved numerically using the finite element method. After using $L$, $d, U, d U / L, \mu U L / d^{2}, \sigma_{l g}$ and $\rho_{(0)}^{s}$ as scales for the horizontal coordinate, vertical coordinate, tangential velocities, vertical velocity, pressure, surface tension and surface density, respectively, one has that the problem is specified by following non-dimensional parameters:

$$
\begin{gathered}
r=\frac{d}{L}, \bar{a}=\frac{a}{L}, \quad R e=\frac{\rho U d}{\mu}, C a=\frac{\mu U}{\sigma_{l g}}, \epsilon=\frac{U \tau}{d}, \bar{\beta}=\frac{\beta U d}{\sigma_{l g}}, \\
Q=\frac{\rho_{(0)}^{s}}{\rho U \tau}, \bar{\alpha}=\frac{\alpha \sigma_{l g}}{U d}, \bar{l}=\frac{l}{L}, \lambda=\frac{\gamma \rho_{(0)}^{s}}{\sigma_{l g}}, \theta_{i} \quad(i=1,2) .
\end{gathered}
$$

As the interface formation theory is applied here without any ad hoc alterations, we may take estimates for the theory's phenomenological constants from independent experiments. Dynamic wetting experiments 11, for example, suggest that for a fluid with $\mu \sim 10 \mathrm{~g} \mathrm{~cm}^{-1} \mathrm{~s}^{-1}$ and $\rho \sim 1 \mathrm{~g} \mathrm{~cm}^{-3}$ we have $\alpha \sim 10^{-8} \mathrm{~g}^{-1} \mathrm{~cm}^{2} \mathrm{~s}, \beta \sim 10^{8} \mathrm{~g} \mathrm{~cm}^{-2} \mathrm{~s}^{-1}, \rho_{(0)}^{s} \sim 10^{-7} \mathrm{~g} \mathrm{~cm}^{-2}$ and $\tau \sim 10^{-7}$ s. Using these estimates and taking typical values associated with the flows of thin liquid films, $U \sim 10 \mathrm{~cm} \mathrm{~s}^{-1}, d \sim 10^{-5} \mathrm{~cm}, \sigma_{l g} \sim 10^{2}$ dyn $\mathrm{cm}^{-1}$ and $l \sim 5 \times 10^{-7} \mathrm{~cm}$, we have the following typical magnitudes of the non-dimensional groups: $R e=10^{-5}, C a=1, \epsilon=10^{-1}$, $\bar{\beta}=10^{2}, Q=10^{-1}, \bar{\alpha}=10^{-2}, \bar{l}=5 \times 10^{-2}$ and $\lambda=2$.

Consider each stripe of solid to have equal width, $a=1 / 2$, and the aspect ratio $r=1$. Then, without loss of generality, we may consider solid 1 to be more hydrophilic than solid 2. Streamlines for the typical values and $\theta_{1}=10^{\circ}, \theta_{2}=80^{\circ}$ are shown in Fig. 2 The less hydrophilic substrate appears to acts as an obstacle to motion with streamlines coming out 

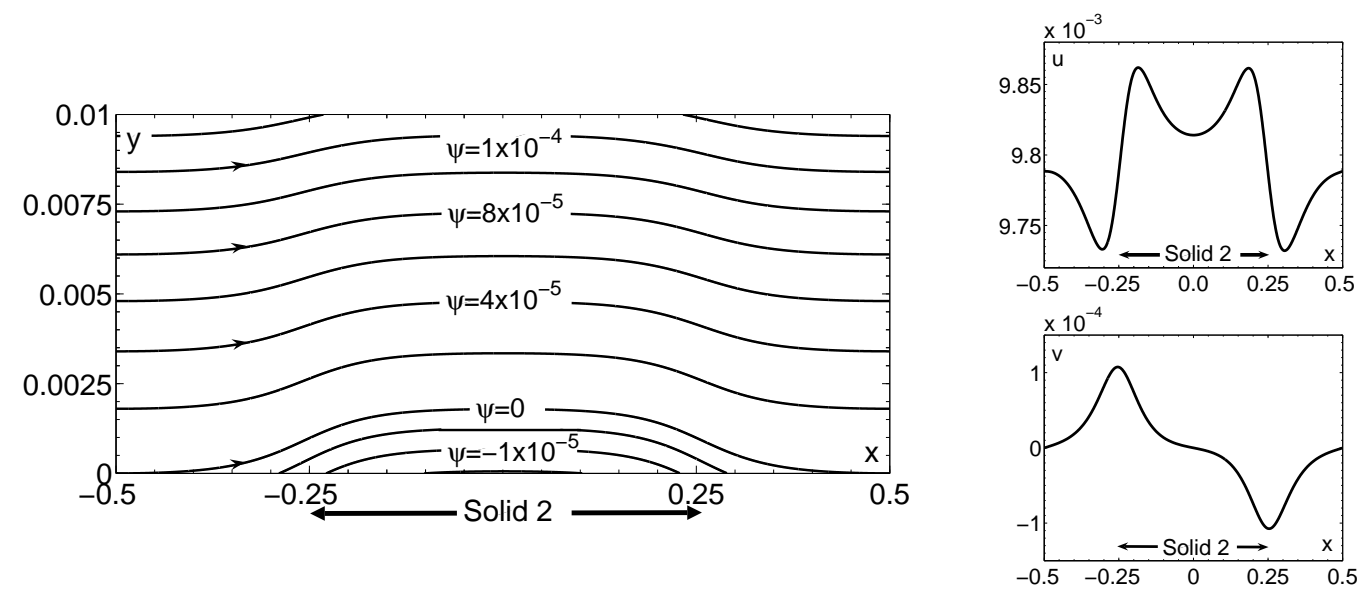

Fig. 2. Streamlines and the corresponding components of velocity on the substrate for flow over a intermittently chemically patterned surface in which values of the streamfunction $\psi$ are given. Parameters are at their typical values with $\theta_{1}=10^{\circ}$ and $\theta_{2}=80^{\circ}$.

of the surface as the solid becomes less hydrophilic, at $x=-0.25$, and entering again as the solid becomes more hydrophilic again, at $x=0.25$. This effect can be seen clearly by looking at the normal velocity on the liquid facing side of the liquid-solid interface in Fig. 2 One can see that the components of velocity are trying to relax to their equilibrium values, $\mathbf{u}_{e}=\left(9.8 \times 10^{-3}, 0\right)$ but are unable to obtain them before the next transition in wettability. This means that the distance between transitions is of the same order as the relaxation length, which is the distance that it takes for surface properties to relax to their equilibrium values. The width of the transition region $\bar{l}$ is far smaller than the relaxation length and, as in [7, its exact size has very little effect on the overall flow field.

Notably, in contrast to studies of slip on super hydrophobic substrates, where the presence of nanobubbles leads to the effective slip length of the system becoming a relevant measure of the effects of chemically patterning the substrate [12 13, here the slip coefficient $\beta$ remains the same on both solids. Importantly, as shown in 7, slip results primarily from the disturbance of the force balance in the liquid-solid interface and not from the tangential stress, as follows from the standard Navier condition. This has significant consequences when attempting to interpret the results of molecular dynamics simulations and experiments in terms of a continuum theory.

Acknowledgement. The authors kindly acknowledge the financial support of Kodak European Research and the EPSRC via a Mathematics CASE award.

\section{References}

1. P. Tabeling, Introduction to Microfluidics (Oxford University Press, Oxford 2006).

2. E. Lauga, M.P. Brenner and H.A. Stone, Handbook of experimental fluid dynamics (Springer, New York 2005).

3. P.A. Thompson and S.M. Troian, Nature 389, (1997) 360-362.

4. C. Cottin-Bizonne, B. Cross, A. Steinberger and E. Charlaix, Phys. Rev. Lett. 94, (2005) 056102.

5. C.L.M.H. Navier, Mém. de l'Acad. de Sciences l'Inst. de France 6, (1823) 389-440.

6. V.P. Sokhan, D. Nicholson and N. Quirke, J. Chem. Phys. 115, (2001) 3878-3887.

7. J.E. Sprittles and Y.D. Shikhmurzaev, Phys. Rev. E. 76, (2007) 021602.

8. Y.D. Shikhmurzaev, Capillary Flows with Forming Interfaces (Taylor \& Francis, London, 2007).

9. Y.D. Shikhmurzaev, IMA J. Appl. Maths, 70, (2005) 880-907.

10. Y.D. Shikhmurzaev, Physica D, 217, (2006) 121-133.

11. T.D. Blake and Y.D. Shikhmurzaev, J. Coll. Int. Sci. 253, (2002) 196-202.

12. E. Lauga and H.A. Stone, J. Fluid Mech. 489, (2003) 55-77.

13. N.V. Priezjev, A.A. Darhuber and S.M. Troian, Phys. Rev. E 71, (2005) 041608. 